\title{
On the decomposition map for symmetric groups
}

\author{
Karin Erdmann
}

Received: 13 November 2007 / Accepted: 20 March 2008 / Published online: 3 April 2008

(C) Springer Science+Business Media, LLC 2008

\begin{abstract}
Let $R^{d}$ be the $\mathbb{Z}$-module generated by the irreducible characters of the symmetric group $\mathcal{S}_{d}$. We determine bases for the kernel of the decomposition map. It is known that $R^{d} \otimes_{\mathbb{Z}} F$ is isomorphic to the radical quotient of the Solomon descent algebra when $F$ is a field of characteristic zero. We show that when $F$ has prime characteristic and $I_{b r}^{d}$ is the kernel of the decomposition map for prime $p$ then $R^{d} / I_{b r}^{d} \otimes_{\mathbb{Z}} F$ is isomorphic to the radical quotient of the $p$-modular Solomon descent algebra.
\end{abstract}

Keywords Representations of symmetric groups · Decomposition map ·

Characters: Brauer characters · Symmetric functions · Solomon descent algebra

Mathematics Subject Classification (2000) 20C20 - 20C30 - 05E99 · 16S99

\section{Introduction}

A basic problem in representation theory of symmetric groups is to express irreducible characters in terms of irreducible Brauer characters on p-regular elements for $p$ a prime number, that is to find the decomposition numbers.

If $G$ is the symmetric group $\mathcal{S}_{d}$ let $R^{d}$ be the $\mathbb{Z}$-module generated by the irreducible characters of $G$, and let $R_{b r}^{d}$ be the $\mathbb{Z}$-module generated by the irreducible Brauer characters of $G$. Restriction of functions to $p$-regular elements induces a $\mathbb{Z}$-homomorphism $\xi: R^{d} \rightarrow R_{b r}^{d}$; and this is known to be surjective. The decomposition numbers describe the kernel of this map, in terms of the bases of ir-

To the memory of Manfred Schocker.

K. Erdmann $(\bowtie)$

Mathematical Institute, 24-29 St. Giles, Oxford OX1 3LB, UK

e-mail: erdmann@maths.ox.ac.uk 
reducible characters and the irreducible Brauer characters. However, although the irreducible Brauer characters have nice parametrizations, they are not known in general (and finding all decomposition numbers is known to be as hard as the Lusztig conjecture for type $A,[8])$.

Therefore one asks whether there are different ways to describe the kernel of the decomposition map, for which explicit answers can be given. The aim of this paper is to give such a description. As an application, it gives a new characterisation of the radical quotient for the Solomon descent algebra.

Finding bases for the kernel of the decomposition map is done via symmetric functions. In this setup, it is equally good to replace the prime $p$ by any positive integer $r>1$. Let $R=\oplus_{d \geq 0} R^{d}$, this is a ring which is identified with the ring $\Lambda$ of symmetric functions via the characteristic map [10, I.7]. Define $\xi$ by restricting class functions to the set of $r$-regular elements; we are interested in the kernel of $\xi$ which we denote by $I_{b r}\left(R^{d}\right)$, or rather in the corresponding subgroup of $\Lambda^{d}$, denoted by $I_{b r}\left(\Lambda^{d}\right)$. Let $\psi^{r}: \Lambda \rightarrow \Lambda$ be the ring homomorphism which takes the variable $x_{i} \in \Lambda$ to $x_{i}^{r}$. Any product $\psi^{r}(f) \cdot g$ of degree $d$ where $f$ has zero constant term belongs to $I_{b r}\left(\Lambda^{d}\right)$; and we obtain $\mathbb{Z}$-bases consisting of functions of this form. This uses results by J. Nuttall [11] and G. Walker [17]. In particular this gives presentations for the Abelian groups $R_{b r}^{d}$. These are given in terms of characteristic zero information, so we conclude that the problem of describing the kernel of the decomposition map for symmetric groups is easier than the problem of finding decomposition numbers.

The direct sum $I:=\oplus_{d \geq 0} I_{b r}\left(\Lambda^{d}\right)$ is an ideal of $\Lambda$, and this gives a presentation for the factor ring, and hence of the ring $R_{b r}=\oplus_{d \geq 0} R_{b r}^{d}$. We obtain that $I$ is the ideal generated by the image $\psi^{r}\left(\Lambda^{+}\right)$of symmetric functions $\Lambda^{+}$with zero constant term. In case $r=p$ prime, the map $\psi^{p}$ may be thought of as a 'Frobenius twist'; on the level of symmetric polynomials it takes the formal character of a module for the general linear group to the formal character of its Frobenius twist. So in this case one could say, roughly speaking, that the kernel of the decomposition map for symmetric groups is determined by the Frobenius twist.

For a fixed $d$, the product of characters induces a ring structure on $R^{d}$ and also on $R_{b r}^{d}$. We take the integral form of $R^{d}$ given by Young characters, then for $F$ a field of characteristic zero, $R^{d} \otimes_{\mathbb{Z}} F$ is isomorphic to the radical quotient of the Solomon descent algebra $\mathcal{D}_{d}$, this was proved in [16]. When $F$ is a field of prime characteristic $p>0$, the radical quotient of the $p$-modular Solomon descent algebra was determined in [2], and also in [3]. Namely, it is isomorphic to the $F$-algebra obtained by reducing the Young characters modulo $p$. We show that this radical quotient is isomorphic to $R_{b r}^{d} \otimes_{\mathbb{Z}} F$. In particular this also gives information on the algebra structure of $R^{d} \otimes_{\mathbb{Z}} F$. Namely, it is the quotient of the descent algebra obtained by base change from the characteristic zero radical quotient.

Part of this existed as a preprint a while ago. In the meantime, one aspect was used in [5] 4.4, and extended by supplying a hard calculation. Subsequently I learned through Manfred Schocker the connection with the Solomon descent algebra, and the last part of this note is only very recent. 


\section{Preliminaries}

2.1 Let $\Lambda^{+}(n, d)$ be the set of all partitions of $d$ with at most $n$ parts. If $\lambda=$ $\left(\lambda_{1}, \lambda_{2}, \cdots, \lambda_{n}\right)$ is a partition then $|\lambda|$ is the sum of the parts. Moreover, if $r$ is a positive integer, then $r \lambda$ is the partition $\left(r \lambda_{1}, r \lambda_{2}, \cdots, r \lambda_{n}\right)$. We also write $\lambda^{r}$ for the partition whose parts are the $\lambda_{i}$, each of them $r$ times. If $\lambda, \mu$ are partitions we denote by $\lambda \cup \mu$ the partition whose parts are those of $\lambda$ and $\mu$ (abusing notation). We denote by $\leq$ the lexicographic order of partitions. A partition $\lambda$ is $r$-regular if it does not have $r$ or more equal parts. We write $\lambda \sim_{r} \emptyset$ if the $r$-core of $\lambda$ is $\emptyset$.

2.2 Let $R=\oplus_{d \geq 0} R^{d}$ where $R^{d}$ is the Abelian group of generalized characters of the symmetric group $\mathcal{S}_{d}$. Then $R$ is a ring which is isomorphic to the ring $\Lambda$ of symmetric functions. That is, $\Lambda=\lim \Lambda_{(n)}$, where $\Lambda_{(n)}$ is the ring of symmetric polynomials in $n$ variables, and where the limit is taken via the $\mathbb{Z}$-linear maps $\Lambda_{(n)} \rightarrow \Lambda_{(m)}$ for $n \geq m$ defined by

$$
x_{i} \rightarrow \begin{cases}x_{i} & i \leq m \\ 0 & i>m\end{cases}
$$

Let $(-,-)$ be the inner product on $R$ defined from the usual scalar product on $R^{d}$ and making $R^{d}$ 's for different $d$ orthogonal. Let also $(-,-)$ be the scalar product on $\Lambda$ such that the Schur functions $s_{\lambda}$ form an orthonormal basis. Then the characteristic map ch : $R \rightarrow \Lambda$ is an isometric ring isomorphism [10] I(7.3).

2.3 We fix an integer $r \geq 1$. There is a ring homomorphism $\psi^{r}: \Lambda \rightarrow \Lambda$ which takes $x_{i}$ to $x_{i}^{r}$; and it is compatible with the projections $\Lambda \rightarrow \Lambda_{(n)}$; see [17], [7]. Consider the case $r=p$ where $K$ is an infinite field of characteristic $p$. If $M$ is a $G L_{n}(K)$ module with formal character $\chi$ then $\psi^{p}(\chi)$ is the formal character of $M^{F}$ where $F$ is the Frobenius map.

2.3.1 By transport of structure, we get a ring homomorphism on $R$ which we also denote by $\psi^{r}$. Suppose $\chi$ is a generalised character of $\mathcal{S}_{d}$ where $d \geq 1$; then via the characteristic isomorphism we obtain the following explicit formula for $\psi^{r}(\chi)$, namely we have for $g \in \mathcal{S}_{d}$

$$
\psi^{r}(\chi)(g)= \begin{cases}z_{r \mu} z_{\mu}^{-1} \chi\left(w_{\mu}\right) & \text { if } g \text { is conjugate to } w_{r \mu} \\ 0 & \text { otherwise }\end{cases}
$$

Here $w_{\lambda}$ is an element of cycle type $\lambda$, and $z_{\lambda}$ is the order of the centralizer of $w_{\lambda}$ in $\mathcal{S}_{d}$. Following [9], an element $g \in \mathcal{S}_{d}$ is said to be $r$-element if each non-trivial cycle has length divisible by $r$, and $g$ is $r$-singular if at least one cycle of $g$ is divisible by $r$. Furthermore, $g$ is $r$-regular if no cycle of $g$ has length divisible by $r$. If $r=p$ is prime, then this is the usual definition of a $p$-regular element of a group.

With this we have seen that $\psi^{r}(\chi)$ is supported on fixed point free $r$-elements of $\mathcal{S}_{d}$. 
2.3.2 The map $\psi^{r}$ has an adjoint with respect to $(-,-)$, denoted by $\psi_{r}$, which is also a ring homomorphism of $\Lambda$ and is compatible with the projections; this is a result in [17]. The map $\psi_{r}$ can be defined as follows, on the complete symmetric functions $h_{d}$. Let $d \geq 1$, then

$$
\psi_{r}\left(h_{d}\right)= \begin{cases}h_{s} & \text { if } d=r s \\ 0 & \text { otherwise }\end{cases}
$$

By transport of structure we get a ring homomorphism of $R$ which we also denote by $\psi_{r}$.

2.4 The following formula is due to J. Nuttall [11]. Let $\omega=e^{2 \pi i / r} \in \mathbb{C}$; then we have for $d \geq 1$

$$
(*) \quad \psi^{r} \psi_{r}\left(h_{d}\right)=\sum_{\alpha \in \Lambda^{+}(r, d)} m_{\alpha}\left(1, \omega, \cdots, \omega^{r-1}\right) h_{\alpha}
$$

where $m_{\alpha}$ is the monomial symmetric function; and $h_{\alpha}=\prod_{i=1}^{r} h_{\alpha_{i}}$ if $\alpha=\left(\alpha_{1}, \alpha_{2}\right.$, $\cdots, \alpha_{r}$ ). (This can be proved as follows. If $H(t)$ is the generating function for the complete symmetric functions, then

$$
\psi^{r} \psi_{r}(H(t))=\prod_{i \geq 0}\left(1-x_{i}^{r} t^{r}\right)^{-1}
$$

see [17] 2.1. Now one observes that $\left(1-\left(x_{i} t\right)^{r}\right)=\prod_{k=0}^{r-1}\left(1-\omega^{k}\left(x_{i} t\right)\right)$, and one uses [10] I(4.2).) Hence for any integer $s \geq 1$ we have

$$
(* *) \quad \psi^{r}\left(h_{s}\right)=\sum_{\alpha \in \Lambda^{+}(r, r s)} m_{\alpha}\left(1, \omega, \cdots, \omega^{r-1}\right) h_{\alpha}
$$

This can also be expressed in terms of Schur functions. Let $s_{\alpha}$ denote the Schur function corresponding to the partition $\alpha$. By [10] I(4.3) and [10] ex. 17 p.50 we have

$$
\psi^{r}\left(h_{s}\right)=\sum_{\alpha \in \Lambda^{+}(r, r s), \alpha \sim{ }_{r} \emptyset} \sigma_{r}(\alpha) s_{\alpha}
$$

Here $\sigma_{r}(\alpha)$ is the sign of the unique permutation $w \in \mathcal{S}_{r}$ such that $\alpha+\delta_{r} \equiv w \delta_{r}(\bmod r)$, where $\delta_{r}=(r-1, r-2, \cdots, 1,0)$.

2.5 Let $\omega$ be as above a primitive complex $r$-th root of unity. We set

$$
z:=\left(1, \omega, \omega^{2}, \cdots, \omega^{r-1}\right)
$$

The values $m_{\alpha}(z)$ where $\alpha \in \Lambda^{+}(r, d)$ are calculated explicitly in [5]4.4. Here we only need a small amount of information which is not hard to obtain directly. The numbers $m_{\alpha}(z)$ are integers, and if $r$ does not divide $d$ then $m_{\alpha}(z)=0$. This follows for example from 2.4 . 
Assume now that $r$ divides $d$, say $d=r s$. Let $m_{i}$ be the multiplicity of $i$ as a part of $\alpha$ so that $\alpha=\left(\cdots, i^{m_{i}}, \cdots, 1^{m_{1}}, 0^{m_{0}}\right)$, with $m_{i} \geq 0$ and $\sum m_{i}=r$. Let $q$ be the greatest common divisor of the $m_{i}$. Then one shows that $m_{\alpha}(z)$ is divisible by $r / q$. (For example, take $m_{\alpha}$ with $r$ variables, then the terms form one orbit under the action of the symmetric group $\mathcal{S}_{r}$ given by change of variables. Analysing this action gives the required answer). One deduces:

2.5.1 We have $m_{\alpha}(z)= \pm 1$ if and only if $\alpha=\left(s^{r}\right)$.

\section{On $\mathbb{Z}$-bases of $\boldsymbol{I}_{b r}$}

3.1 We fix an integer $r>1$. For a character $\chi \in R^{d}$, let $\xi(\chi)$ denote the restriction of $\chi$ to the set of $r$-regular elements of $\mathcal{S}_{d}$. Let $R_{b r}^{d}$ be the $\mathbb{Z}$-module generated by the $\xi(\chi)$, a submodule of the $\mathbb{Z}$-valued class functions on $r$-regular elements (see 2.3.1). When $r=p$ is prime then $R_{b r}^{d}=\sum_{\lambda} \mathbb{Z} \beta^{\lambda}$, here the $\beta^{\lambda}$ are the irreducible Brauer characters, which can be labelled by $p$-regular partitions of $d$. For a discussion of the decomposition map for finite groups in general, we refer to [15].

We have then a surjective $\mathbb{Z}$-homomorphism $\xi: R^{d} \rightarrow R_{b r}^{d}$. For arbitrary $r$, it is proved in [9]4.2 that the set $\xi\left(\chi^{\lambda}\right)$ for $\lambda r$-regular, is a free $\mathbb{Z}$ basis for $R_{b r}^{d}$. [Here $\chi^{\lambda}$ is the irreducible character of $\mathcal{S}_{d}$ corresponding to partition $\lambda$, as usual.]

Let $I_{b r}\left(R^{d}\right)$ denote the kernel of $\xi$, and define

$$
R_{b r}=\oplus_{d \geq 0} R_{b r}^{d}, \quad I_{b r}(R)=\oplus_{d \geq 0} I_{b r}\left(R^{d}\right) .
$$

We have also a $\mathbb{Z}$-homomorphism $R \rightarrow R_{b r}$ which we denote by $\xi$, extending the homogeneous restriction maps. Its kernel is $I_{b r}(R)$ which is an ideal, and $R_{b r}$ has a ring structure such that $\xi$ is a ring homomorphism. We are interested in $I_{b r}\left(R^{d}\right)$ and $I_{b r}(R)$; we will study their images under the characteristic isomorphism in $\Lambda$, which we denote by $I_{b r}\left(\Lambda^{d}\right)$ and $I_{b r}(\Lambda)$.

Let $\lambda=\left(\lambda_{1}, \cdots, \lambda_{n}\right)$ be a partition of $d$. The symmetric function $h_{\lambda}=\prod_{i=1}^{n} h_{\lambda_{i}}$ corresponds under the characteristic isomorphism to the character $\varphi^{\lambda}$ of the permutation module on the cosets of the Young subgroup $\mathcal{S}_{\lambda_{1}} \times \cdots \times \mathcal{S}_{\lambda_{n}}$ [10]I.7; we call the $\varphi^{\lambda}$ Young characters. Moreover, the Schur function $s_{\lambda}$ corresponds to the irreducible character $\chi^{\lambda}$ of $\mathcal{S}_{d}[10] \mathrm{I}(7.5)$.

3.1.1 The observation in 2.3.1 shows that $I_{b r}(R)$ contains $\psi^{r}\left(R^{d}\right)$ for $d \geq 1$, and then as well $\psi^{r}\left(R^{d}\right) R$. Hence $I_{b r}(\Lambda)$ contains $\psi^{r}\left(\Lambda^{d}\right) \Lambda$, for $d \geq 1$.

3.2 First we give a $\mathbb{Z}$-basis of $I_{b r}(\Lambda)$ in terms of the symmetric functions $h_{\lambda}$.

Theorem Let $r \geq 1$. Then $\Lambda$ has $\mathbb{Z}$-basis

$$
\mathcal{H}_{r}:=\left\{\psi^{r}\left(h_{\lambda}\right) \cdot h_{\mu}: \lambda, \mu \text { partitions with } \mu \text { r-regular }\right\} .
$$

Moreover if $r>1$ then $I_{b r}(\Lambda)$ has $\mathbb{Z}$-basis $\mathcal{H}_{r}^{0}:=\left\{\psi^{r}\left(h_{\lambda}\right) \cdot h_{\mu} \in \mathcal{H}_{r}: \lambda \neq \emptyset\right\}$. 
We remark that $\mathcal{H}_{1}$ is one of the usual bases for $\Lambda$ [10] $\mathrm{I}(2.8)$. For the proof we will use the following.

3.3 Suppose $\gamma$ is a partition such that $h_{\gamma}$ occurs with non-zero coefficient in $\psi^{r}\left(h_{\lambda}\right)$. $h_{\mu}$, then $\gamma \geq \lambda^{r} \cup \mu$.

Proof By the hypothesis there are partitions $\tau^{(i)}$ such that $h_{\tau^{(i)}}$ occurs in $\psi^{r}\left(h_{\lambda_{i}}\right)$ and $\gamma=\tau^{(1)} \cup \tau^{(2)} \cup \cdots \cup \tau^{(l(\lambda))} \cup \mu$. Then $\left|\tau^{(i)}\right|=r\left|\lambda_{i}\right|$ and $\tau^{(i)}$ has at most $r$ parts. Now, $\left(\lambda_{i}^{r}\right)$ is the least partition of $r\left|\lambda_{i}\right|$ with at most $r$ parts, so $\tau^{(i)} \geq\left(\lambda_{i}^{r}\right)$ for each $i$. So we must show the following.

If $\tau^{(i)}, \delta^{(i)}$ are partitions with $\tau^{(i)} \geq \delta^{(i)}$ and $\left|\tau^{(i)}\right|=\left|\delta^{(i)}\right|$ for all $i$ then $\tau^{(1)} \cup$ $\tau^{(2)} \cup \cdots \cup \tau^{(m)} \geq \delta^{(1)} \cup \delta^{(2)} \cup \cdots \cup \delta^{(\bar{m})}$.

Let $c_{1}$ be the first part of $\tau^{(1)} \cup \tau^{(2)} \cup \cdots \cup \tau^{(m)}$. Then $c_{1} \geq\left(\tau^{(i)}\right)_{1}$ for each $i$, and so $c_{1} \geq\left(\delta^{(i)}\right)_{1}$ for each $i$. If $c_{1}$ is greater than the first part of $\delta^{(1)} \cup \delta^{(2)} \cup \cdots \cup \delta^{(m)}$ then we are done. Otherwise, there is some $j$ with $\left(\delta^{(j)}\right)_{1}=c_{1}$, and then $\left(\tau^{(j)}\right)_{1}=c_{1}$ as well. Remove the first part from $\tau^{(j)}$ and from $\delta^{(j)}$. The induction hypothesis gives the statement.

3.4 We will now prove 3.2, by considering $\mathcal{H}_{r} \cap \Lambda^{d}$ for a fixed degree $d$. We know that $\Lambda^{d}$ has a $\mathbb{Z}$-basis contained in $\mathcal{H}_{1}$; and so it suffices to show that, the coefficient matrix $M\left(\mathcal{H}_{r}, \mathcal{H}_{1}\right)$ is lower triangular, with diagonal entries \pm 1 . To do so, we label the symmetric function $\psi^{r}\left(h_{\lambda}\right) \cdot h_{\mu}$ by the partition $\lambda^{r} \cup \mu$; and we order these lexicographically.

If $h_{\gamma}$ occurs in $\psi^{r}\left(h_{\lambda}\right) \cdot h_{\mu}$ then $\gamma \geq \lambda^{r} \cup \mu$; this follows from 3.3. Hence the coefficient matrix is lower triangular. Moreover, if $\gamma=\lambda^{r} \cup \mu$ then $\tau^{(i)}=\left(\lambda_{i}^{r}\right)$ and it follows that the coefficient of $h_{\gamma}$ in $\psi^{r}\left(h_{\lambda}\right) h_{\mu}$ is equal to \pm 1 , by 2.5.1. Note also that the rank of $\Lambda^{d}$ is the same as the number of elements in $\mathcal{H}_{r} \cap \Lambda^{d}$. Hence we are done with the first part.

Consider the second part, by 3.1.1 we know that $\mathcal{H}_{r}^{0} \cap \Lambda^{d}$ is contained in $I_{b r}\left(\Lambda^{d}\right)$, and by the first part it is $\mathbb{Z}$-linearly independent. We know that the image of $\xi$ is free over $\mathbb{Z}$ of rank equal to the number of $r$-regular partitions of $d$. Hence the kernel of $\xi$ has rank equal to the size of $\mathcal{H}_{r}^{0} \cap \Lambda^{d}$, and this implies that $\mathcal{H}_{r}^{0} \cap \Lambda^{d}$ is a $\mathbb{Z}$-basis for $I_{b r}\left(\Lambda^{d}\right)$.

\subsection{Theorem Let $r \geq 1$. Then $\Lambda$ has $\mathbb{Z}$-basis}

$$
\Sigma_{r}:=\left\{\psi^{r}\left(s_{\lambda}\right) \cdot s_{\mu}: \lambda, \mu \text { partitions with } \mu \text { r-regular }\right\} .
$$

Moreover if $r>1$ then $I_{b r}(\Lambda)$ has $\mathbb{Z}$-basis $\left\{\psi^{r}\left(s_{\lambda}\right) \cdot s_{\mu} \in \Sigma_{r}: \lambda \neq \emptyset\right\}$.

Proof We consider $\Sigma_{r} \cap \Lambda^{d}$ for a fixed degree $d$. For the first part, we will show that the coefficient matrix $M\left(\Sigma_{r}, \mathcal{H}_{r}\right)$ is lower triangular, with diagonal entries \pm 1 . We label $\psi^{r}\left(s_{\lambda}\right) \cdot s_{\mu}$ and also $\psi^{r}\left(h_{\lambda}\right) \cdot h_{\mu}$ by the partition associated to $\lambda^{r} \cup \mu$, and we order these lexicographically, as before.

Consider $\psi^{r}\left(s_{\lambda}\right) \cdot s_{\mu}$. Express $s_{\lambda}$ in terms of the $h_{\gamma}$; the transition matrix is $M(s, h)=K^{*}$, in the notation of [10] $\mathrm{I}(6.3)$, and it is strictly lower unitriangular. Express also $s_{\mu}$ in terms of the basis $\mathcal{H}_{r}$, the transition matrix is the product of $M(s, h)$ 
and $M\left(\mathcal{H}_{1}, \mathcal{H}_{r}\right)$, so by [10] $\mathrm{I}(6.3)$ and 2.4 it is lower triangular, with diagonal entries \pm 1 . We get

$$
\text { (*) } \psi^{r}\left(s_{\lambda}\right) \cdot s_{\mu}=\left(\sum_{\lambda \leq \gamma} K_{\lambda \gamma}^{*} \psi^{r}\left(h_{\gamma}\right)\right) \cdot\left(\sum b_{\mu, \beta^{r} \cup \alpha} \psi^{r}\left(h_{\beta}\right) \cdot h_{\alpha}\right)
$$

the second sum is taken over all $\alpha, \beta$ such that $\mu \leq \beta^{r} \cup \alpha$ and $\alpha$ is $r$-regular. Replacing $\psi^{r}\left(h_{\gamma}\right) \cdot \psi^{r}\left(h_{\beta}\right)$ by $\psi^{r}\left(h_{\gamma \cup \beta}\right)$ and re-arranging gives the expression of $\psi^{r}\left(s_{\lambda}\right) \cdot s_{\mu}$ in terms of the basis $\mathcal{H}_{r}$. We are done if we show that the coefficient matrix is unitriangular with \pm 1 's in the diagonal.

We claim that the coefficient of $\psi^{r}\left(h_{\lambda}\right) \cdot h_{\mu}$ in this expression is \pm 1 . The coefficient is equal to $\sum K_{\lambda \gamma}^{*} b_{\mu, \beta^{r} \cup \mu}$ where the sum is taken over all $\gamma, \beta$ such that $\lambda \leq \gamma$, $\mu \leq \beta^{r} \cup \mu$ and $\gamma \cup \beta=\lambda$ These conditions imply $\beta=\emptyset$ and $\lambda=\gamma$, so there is only one term and it is \pm 1 .

Suppose the coefficient of $\psi^{r}\left(h_{\sigma}\right) \cdot h_{\alpha}$ is non-zero, for $\alpha r$-regular. We must show that then $\lambda^{r} \cup \mu \leq \sigma^{r} \cup \alpha$. The coefficient is equal to

$$
\sum K_{\lambda \gamma}^{*} b_{\mu, \beta^{r} \cup \alpha}
$$

where the sum is taken over all $\gamma, \beta$ with $\gamma \cup \beta=\sigma, \lambda \leq \gamma$ and $\mu \leq \beta^{r} \cup \alpha$. Then $\lambda^{r} \leq \gamma^{r}$, hence we deduce

$$
\lambda^{r} \cup \mu \leq \gamma^{r} \cup \mu \leq \gamma^{r} \cup \beta^{r} \cup \alpha=(\gamma \cup \beta)^{r} \cup \alpha=\sigma^{r} \cup \alpha .
$$

The rest follows by the same argument as the second part of 3.4 .

3.6 Corollary Let $r \geq 1$. Then $R$ has $\mathbb{Z}$-bases

$$
\begin{aligned}
\mathcal{H}_{r} & :=\left\{\psi^{r}\left(\eta_{\lambda}\right) \cdot \eta_{\mu}: \lambda, \mu \text { partitions and } \mu \text { r-regular }\right\} \\
\Sigma_{r} & :=\left\{\psi^{r}\left(\chi^{\lambda}\right) \cdot \chi^{\mu}: \lambda, \mu \text { partitions and } \mu \text { r-regular }\right\} .
\end{aligned}
$$

Moreover $I_{b r}(R)$ has $\mathbb{Z}$-bases

$$
\mathcal{H}_{r}^{0}:=\left\{\psi^{r}\left(\eta_{\lambda}\right) \cdot \eta_{\mu} \in \mathcal{H}_{r}: \lambda \neq \emptyset\right\} \quad \text { and } \quad \Sigma_{r}^{0}:=\left\{\psi^{r}\left(\chi^{\lambda}\right) \cdot \chi^{\mu} \in \Sigma_{r}: \lambda \neq \emptyset\right\}
$$

This follows from 3.2 and 3.5.

\section{Some presentations and relations}

4.1 The basis elements of degree $d$ in $\mathcal{H}_{r}$, or $\Sigma_{r}$ respectively describe a complete minimal set of relations for $I_{b r}\left(\Lambda^{d}\right)$ or equivalently for $I_{b r}\left(R^{d}\right)$. The ring $R_{b r}$ is isomorphic to $\Lambda / I$ where $I=I_{b r}(\Lambda)$. The results 3.2 and 3.5 show that $I$ is generated by $\psi^{r}\left(\Lambda^{+}\right)$where $\Lambda^{+}=\oplus_{d \geq 1} \Lambda^{d}$. Hence we get from 2.4 the presentation

$$
R_{b r} \cong \mathbf{Z}\left[h_{1}, h_{1}, \ldots\right] /\left\langle\sum_{\alpha \in \Lambda^{+}(r, r s)} m_{\alpha}(z) h_{\alpha}, s=1,2, \ldots\right\rangle .
$$


An explicit description of the ideal $I$ can be found in [5] 4.4 .

4.2 Assume now $r=p$, we consider the ring $R_{b r}$ modulo $p$. We deduce the following, which is a special case of [6] 4.7, Remark 3.

Corollary Let $F$ be a field of characteristic p. Then the ring $R_{b r} \otimes_{Z} F$ is isomorphic to

$$
F\left[h_{1}, h_{2}, \ldots\right] /\left\langle h_{s}^{p}, s=1,2, \ldots\right\rangle
$$

where the $h_{i}$ are algebraically independent variables.

Proof For each $d \geq 1$ we have an exact sequence of $\mathbb{Z}$-modules

$$
0 \rightarrow I_{b r}\left(\Lambda^{d}\right) \rightarrow \Lambda^{d} \rightarrow \Lambda_{b r}^{d} \rightarrow 0
$$

By 2.2 this is a split exact sequence of free $\mathbb{Z}$-modules. We deduce that $R_{b r}^{d} \otimes F \cong$ $\left(\Lambda^{d} \otimes F\right) /\left(I_{b r}\left(\Lambda^{d}\right) \otimes F\right),\left(\right.$ writing $\left.\otimes=\otimes_{\mathbb{Z}}\right)$ and

$$
R_{b r} \otimes F \cong \oplus_{d \geq 0}\left(\Lambda^{d} \otimes F\right) /\left(I_{b r}\left(\Lambda^{d}\right) \otimes F\right) \cong(\Lambda \otimes F) /\left(I_{b r}(\Lambda) \otimes F\right) .
$$

Moreover $\Lambda \otimes F$ is the free polynomial ring in the independent variables $h_{s} \otimes 1$. We claim now that $I_{b r}(\Lambda) \otimes F$ is equal to the ideal of $\Lambda \otimes F$ generated by $\left(h_{s} \otimes 1\right)^{p}$, $s=1,2, \ldots$. This holds because $I_{b r}(\Lambda)$ is generated by the $\psi^{p}\left(h_{s}\right)$, and by 2.5 we have over characteristic $p$ that

$$
\psi^{p}\left(h_{s}\right) \otimes 1=(-1)^{(p-1) s} h_{\left(s^{p}\right)} \otimes 1=h_{s}^{p} \otimes 1 .
$$

4.3 We can also exploit the basis in 3.5. Since the Schur functions form a $\mathbb{Z}$-basis of $\Lambda$, there are integers $b_{\gamma}$ such that $\psi^{r}\left(s_{\lambda}\right)=\sum_{\gamma} b_{\gamma} s_{\gamma}$. Then we have by 2.3.2 that

$$
b_{\gamma}=\left(\psi^{r}\left(s_{\lambda}\right), s_{\gamma}\right)=\left(s_{\lambda}, \psi_{r}\left(s_{\gamma}\right)\right) \text {. }
$$

Now, $\psi_{r}\left(s_{\gamma}\right)$ has been determined by G. Walker; he proved the following [17] 2.3.

Theorem Suppose $\gamma$ has empty r-core. Then we have

$$
\psi_{r}\left(s_{\gamma}\right)=\sigma_{r}(\gamma) \cdot \prod_{k=0}^{r-1} s_{\gamma}(k)
$$

Here $\gamma(0), \cdots, \gamma(r-1)$ are the r-quotients of $\gamma$. Otherwise $\psi_{r}\left(s_{\gamma}\right)=0$.

We deduce $b_{\gamma}=0$ unless $\gamma \sim_{r} \emptyset$. In this case, $b_{\gamma}=\sigma_{r}(\gamma) L R_{\gamma(0), \cdots, \gamma(r-1)}^{\lambda}$ where $L R_{\tau(0), \cdots, \tau(m)}^{\lambda}$ is the coefficient of $s_{\lambda}$ in the product $s_{\tau(0)} \cdot s_{\tau(1)} \cdots s_{\tau(m)}$. It follows 
that

$$
\psi^{r}\left(s_{\lambda}\right) \cdot s_{\mu}=\sum_{|\alpha|=d}\left(\sum_{\gamma \sim_{r} \emptyset} \sigma_{r}(\gamma) L R_{\gamma(0), \cdots, \gamma(r-1)}^{\lambda} \cdot L R_{\gamma, \mu}^{\alpha}\right) s_{\alpha}
$$

A similar formula was obtained in [1].

4.4 Assume now that $r=p$, a prime. Consider the relations $\psi^{r}\left(\varphi^{\lambda}\right) \cdot \varphi^{\mu}$ with $\mu$ $p$-regular, and $\lambda \neq \varnothing$ for the symmetric groups. Recall that we use the partition $\gamma=\lambda^{p} \cup \mu$ as a label, this is $p$-singular and every $p$-singular partition has a unique expression of this form. We deduce from 3.6:

Corollary For every $p$-singular partition $\gamma$, there is a generalized character $\sum a_{\beta} \varphi^{\beta}$ with $a_{\beta} \in \mathbb{Z}$, unique up to scalar multiples which vanishes on p-regular elements such that $a_{\gamma} \neq 0$ and moreover $a_{\beta} \neq 0$ implies $\gamma \leq \beta$.

There is also a block version for such class function.

\section{Connection with the Solomon descent algebra}

5.1 Let $d$ be a positive integer. We recall some properties of the Solomon descent algebra of the symmetric group $\mathcal{S}_{d}$ (details can be found in [12], [4], [13], [2], [3], [16]). Let $\mu$ be a composition of $d$, and let $\mathcal{S}_{\mu}$ be the associated Young subgroup of $\mathcal{S}_{d}$. Each right coset of $\mathcal{S}_{\mu}$ in $\mathcal{S}_{d}$ contains a unique permutation of minimal length (see for example [16, Lemma 1]). Define $X^{\mu}$ to be the sum in the integral group ring $\mathbb{Z} \mathcal{S}_{d}$ of all these minimal coset representatives. Solomon [16, Theorem 1] proved that the $\mathbb{Z}$-linear span $\mathcal{D}_{d}$ of the elements $X^{\mu}$ ( $\mu$ any composition of $d$ ), is a subring of $\mathbb{Z} \mathcal{S}_{d}$, which is now called the Solomon descent algebra of $\mathcal{S}_{d}$. In fact, he showed that the multiplication constants with respect to the basis of the $X^{\mu}$ are precisely the multiplication constants for the Young characters. Denote for the moment by $\mathcal{C}_{d}$ the $\mathbb{Z}$-span of the Young characters, a subring of the ring of $\mathbb{Z}$-valued class functions of $\mathcal{S}_{d}$. If $\varphi^{\mu}$ is the Young character induced from the trivial character of $\mathcal{S}_{\mu}$, then the $\mathbb{Z}$-linear map $c_{d}: \mathcal{D}_{d} \rightarrow \mathcal{C}_{d}$ which takes

$$
X^{\mu} \longmapsto \varphi^{\mu}
$$

for compositions $\mu$ of $d$, is a surjective ring homomorphism.

Let $F$ be a field, then the $F$-linear span $\mathcal{D}_{d, F}$ of the elements $X^{\mu}$ is a subalgebra of the group algebra $F \mathcal{S}_{d}$, while the $F$-linear span $\mathcal{C}_{d, F}$ of the $F$-valued Young characters

$$
\varphi^{\mu, F}: \mathcal{S}_{d} \rightarrow F, \pi \mapsto \varphi^{\mu}(\pi) \cdot 1_{F}
$$

is a subalgebra of the algebra of $F$-valued class functions of $\mathcal{S}_{d}$. The $F$-linear map $c_{d, F}: \mathcal{D}_{d, F} \rightarrow \mathcal{C}_{d, F}$ taking $X^{\mu}$ to $\varphi^{\mu, F}$ for compositions $\mu$ of $d$, is an epimorphism of algebras. Moreover, 
Theorem The kernel of $c_{d, F}$ is the radical of $\mathcal{D}_{d, F}$.

This is [16] Theorem 3 for characteristic zero, and it is [2] Theorem 2 for prime characteristic. Hence $\mathcal{C}_{d, F}$ is isomorphic to the radical quotient $\mathcal{D}_{d, F} / \operatorname{rad} \mathcal{D}_{d, F}$.

5.2 We will relate this to the decomposition map. First, we give a different basis for $R_{b r}^{d}$. We know that $R_{b r}^{d}$ has free $\mathbb{Z}$-basis the $\xi\left(\chi^{\lambda}\right)$ for $\lambda$ p-regular. Let $\varphi^{\lambda}$ be the Young character associated to $\lambda$ and let $\varphi_{0}^{\lambda}$ be its restriction to $p$-regular elements (that is the image of $\varphi^{\lambda}$ under the decomposition map).

Lemma The set $\mathcal{B}:=\left\{\varphi_{0}^{\lambda}: \lambda\right.$ a $p$-regular partition of $\left.d\right\}$ is a $\mathbb{Z}$-basis for $R_{b r}^{d}$.

This holds because the coefficient matrix obtained by expressing $\varphi^{\lambda}$ in terms of $\chi^{\mu}$ is lower unitriangular.

We work now with the $\mathbb{Z}$-bases of $R^{d}$ and $R_{b r}^{d}$ given by $\varphi^{\lambda}$ and the $\varphi_{0}^{\lambda}$ respectively. We view $R^{d}$ as a ring with respect to the multiplication of characters. The decomposition map $\xi$ is then a ring homomorphism and hence $I_{b r}^{d}$ is an ideal of $R^{d}$.

Theorem 1 Assume $F$ has characteristic $p$. Then $R_{b r}^{d} \otimes_{\mathbb{Z}} F$ is isomorphic to the radical quotient $\mathcal{C}_{d, F}$ of the Solomon descent algebra $\mathcal{D}_{d, F}$.

Proof The ring $R^{d}$ is the same as $\mathcal{C}_{d}$. We have therefore two surjective $F$-algebra maps starting at $\mathcal{C}_{d} \otimes F$. One is the map which takes $\varphi^{\lambda} \otimes 1$ to $\varphi^{\lambda, F}$, call the map $\rho$. The other is $\xi \otimes 1$. Furthermore, the images of these, that is $\mathcal{C}_{d, F}$ and $R_{b r}^{d} \otimes F$ are $F$ vector spaces of the same dimension. So it is enough to show that $\rho$ maps the $I_{b r} \otimes F$, that is the kernel of $\xi \otimes 1$, to zero. This will induce a surjection from $R_{b r}^{d} \otimes F$ onto $\mathcal{C}_{d, F}$, which must be an isomorphism, by dimensions.

We use the basis from 3.6. It suffices to show that any $\psi^{p}\left(\varphi^{\lambda}\right) \varphi^{\mu}$ with $\lambda \neq \emptyset$ (and of the right degree $d=p|\lambda|+|\mu|)$ lies in the kernel of $\rho$, that is, all its values are divisible by $p$.

We have seen in 2.3.1 that the class function $\chi_{1}:=\psi^{p}\left(\varphi^{\lambda}\right)$ is supported on fixed point free elements for which all cycles have lengths divisible by $p$. Let $\chi_{2}=\phi^{\mu}$, then

$$
\chi_{1} \chi_{2}=\operatorname{Ind}_{H}^{G}\left(\chi_{1} \otimes \chi_{2}\right)
$$

where $H=\mathcal{S}_{m p} \times \mathcal{S}_{t}$ with $m=|\lambda|$ and $t=|\mu|$. Let $\eta=\chi_{1} \otimes \chi_{2}$; then $\operatorname{Ind}_{H}^{G}(\eta)(g)=$ $\sum_{i=1}^{m} \dot{\eta}\left(g_{i} g g_{i}^{-1}\right)$ where $\left\{g_{1}, \ldots, g_{m}\right\}$ are coset representatives of $H$ in $G$ and where

$$
\dot{\eta}(g)= \begin{cases}\eta(g) & g \in H \\ 0 & \text { otherwise }\end{cases}
$$

Suppose $\dot{\eta}\left(y^{-1} g y\right)$ is non-zero. Then $y^{-1} g y=g_{1} g_{2}$ with $g_{1} \in \mathcal{S}_{m p}$ and $g_{2} \in \mathcal{S}_{t}$. Furthermore, $g_{1}$ has cycle type $p \gamma \neq \emptyset$, and we have $\dot{\eta}\left(g_{1} g_{2}\right)=\chi_{1}\left(g_{1}\right) \chi_{2}\left(g_{2}\right)$. By 
2.3.1, the character value $\chi_{1}\left(g_{1}\right)$ is divisible by $p$, and since $\chi_{2}\left(g_{2}\right)$ is an integer we deduce that $\operatorname{Ind}_{H}^{G}(\eta)(g)$ is divisible by $p$ as required.

Corollary The radical quotient of the $F$-algebra $R^{d} \otimes_{\mathbb{Z}} F$ is isomorphic to the radical quotient of the Solomon descent algebra $\mathcal{D}_{d, F}$.

Given an algebra which is not semisimple, then it is a basic problem to understand the algebra modulo the square of its radical, that is to understand its Ext quiver.

Manfred Schocker worked on finding the Ext quiver of descent algebras. Some examples are given in [13]. When $F$ has characteristic zero, he gives a complete answer in [14] and in fact the algebras he studies there are more general. He writes that the quiver for non-zero characteristic is not yet understood. The connection with the decomposition map should be an additional reason why the quiver is interesting, and also perhaps give a different kind of information.

\section{References}

1. Ariki, S., Nakajima, T., Yamada, H.F.: Reduced Schur functions and the Littlewood-Richardson coefficients. J. Lond. Math. Soc. 59, 396-406 (1999)

2. Atkinson, M.D., van Willigenburg, S.: The p-modular descent algebra of the symmetric group. Bull. Lond. Math. Soc. 29, 407-414 (1997)

3. Atkinson, M.D., Pfeiffer, G., van Willigenburg, S.: The p-modular descent algebra. Algebr. Represent. Theory 5, 101-113 (2002)

4. Blessenohl, D., Schocker, M.: Noncommutative Character Theory of the Symmetric Group. Imperial College Press, London (2005)

5. Donkin, S.: The q-Schur algebra. LMS Lecture Notes Series, vol. 253. Cambridge University Press, Cambridge (1998)

6. Donkin, S., Erdmann, K.: Tilting modules, symmetric functions and the module structure of the free Lie algebra. J. Algebra 203, 69-90 (1998)

7. Doty, S., Walker, G.: Modular symmetric functions and irreducible modular representations of general linear groups. J. Pure Appl. Algebra 82, 1-26 (1992)

8. Erdmann, K.: Decomposition numbers for symmetric groups and composition factors of Weyl modules. J. Algebra 180, 316-320 (1996)

9. Külshammer, B., Olsson, J.B., Robinson, G.R.: Generalized blocks for symmetric groups. Invent. Math. 151, 513-552 (2003)

10. MacDonald, I.G.: Symmetric Functions and Hall Polynomials, 2nd edn. Oxford Mathematical Monographs. Clarendon Press, Oxford (1995)

11. Nuttall, J.: Modular symmetric functions and Doty's conjecture. PhD Thesis, University of London (1997)

12. Reutenauer, C.: Free Lie Algebras. London Mathematical Society Monographs (New Series), vol. 7 (1993)

13. Schocker, M.: The descent algebra of the symmetric group. Fields Inst. Commun. 40, 145-161 (2004)

14. Schocker, M.: The module structure of the Solomon-Tits algebra of the symmetric group. J. Algebra 301, 554-586 (2006)

15. Serre, J.-P.: Linear Representations of Finite Groups. Graduate Text in Mathematics, vol. 42. Springer, Berlin (1977)

16. Solomon, L.: A Mackey formula in the group ring of a Coxeter group. J. Algebra 41, 255-268 (1976)

17. Walker, G.: Modular Schur functions. Trans. Am. Math. Soc. 346, 569-604 (1994) 